\title{
Heralded generation of multi-photon entanglement
}

\author{
Philip Walther ${ }^{1, \dagger}$, Markus Aspelmeyer ${ }^{1,2}$, Anton Zeilinger ${ }^{1,2}$ \\ 1 Institute for Experimental Physics, University of Vienna, Boltzmanngasse 5, A-1090 Vienna, Austria \\ 2 Institute for Quantum Optics and Quantum Information (IQOQI), \\ Austrian Academy of Sciences, Boltzmanngasse 3, A-1090 Vienna, Austria \\ $\dagger$ present address: Department of Physics, Harvard University, \\ 17 Oxford Street, Cambridge, MA 02138, USA
}

\begin{abstract}
We present a new scheme, based only on linear optics and standard photon detection, that allows to generate heralded multi-photon entangled states of arbitrary photon number from spontaneous parametric downconversion (PDC) in the weak interaction regime. The scheme also works in the strong interaction regime, i.e. for the production of large photon numbers, when photon-number resolving detectors with nearly perfect quantum efficiency are used. In addition, the same setup can be used for quantum metrology as multi-photon interferometer with sensitivity at the Heisenberg-limit.
\end{abstract}

The controlled generation of single photons and of multi-photon entangled states is at the heart of quantum information processing, in particular quantum cryptography [1, 2] and scalable approaches towards photonicsbased quantum computing schemes $[3,4,5]$. Up to now the best source for heralded single-photon states is spontaneous parametric down-conversion (PDC) [6], in which pairs of strongly time-correlated photons are emitted into two spatial modes and where the detection of one photon of a pair indicates with a high probability the presence of a single-photon in the second mode. Such conditional methods achieve preparation efficiencies of up to $85 \%$ [8] with state qualities of $g^{(2)}(0) \approx 1 \times 10^{-3}$, yet unchallenged by alternative approaches based on atomic or solid states systems. An important goal is to achieve similar heralding for entangled multi-photon states. A problem arises because of the probabilistic emission of PDC. Since pairs of photons are not created 'event-ready' [9], all photons involved in a protocol need to be measured to ensure that the wanted multi-photon state has been created. Several approaches exist to overcome the probabilistic emission in PDC and to prepare two-photon entangled states conditioned on the detection of auxiliary photons $[10,11,12,13,14]$. However, these suggested schemes have either low production probability at high state fidelities for their generated entangled pairs, or they rely on still unavailable detectors that unambiguously resolve photon numbers or on nonlinear interactions between photons, which are too weak to be exploited for applications. We propose a new scheme to generate heralded multi-photon states of arbitrary photon number using only linear optics and standard (non numberresolving) photon detectors. We suggest the heralded generation of $\mathrm{N}$-particle entangled states of the GHZ type, $|\Psi\rangle^{(G H Z)}=1 / \sqrt{2}\left(|0\rangle^{\otimes N}+|1\rangle^{\otimes N}\right)$, from a $2 \mathrm{~N}$ pairemission from PDC. In addition, the setup can be used to generate path-entangled photon-number states of the NOON type, $|\Psi\rangle^{(N O O N)}=1 / \sqrt{(2)}(|4 N\rangle|0\rangle+|0\rangle|4 N\rangle)$, which are of practical relevance for quantum metrology at the Heisenberg limit.
Our proposal is based on separating photon pairs into different pairs of modes and utilizing two-particleinterferometry rather than distinguishing photon numbers or employing nonlinear beamsplitters. A crucial element is the polarizing beam splitter (PBS) that transmits horizontally $(H)$ polarized light and reflects vertically $(V)$ polarized light. This property of the PBS allows 'targeted projections' onto either path-entangled states [15] or polarization-entangled states [16] or a combination of both. Such a successful two-photon interaction can be detected by a two-photon coincidence measurement, where in each output mode one and only one photon is propagating. For the case where two photons arrive from different input modes the PBS acts as parity check [17]. If the input photons propagate within the same mode they must have orthogonal polarizations for being split up into the two different output modes.

Fig. 1 gives a schematic diagram of a possible setup to generate N-particle quantum states of the GHZ type non-destructively, i.e. event-ready. Consider first the case for the heralded generation of the Bell state state $\left|\Phi^{+}\right\rangle=1 / \sqrt{2}\left(|0\rangle^{\otimes 2}+|1\rangle^{\otimes 2}\right)$ (Fig. 1a). An ultra-violet (UV) pulse passes through two co-linearly aligned crystals for PDC, probabilistically producing pairs of energydegenerate polarization-entangled photons into the spatial modes $a 2 \& b 2$ and $a 4 \& b 4$. The UV pulse is reflected back at an adjustable mirror, and can thus also emit entangled photon pairs into the modes $a 1 \& b 1$ and $a 3 \&$ $b 3$. The setup is aligned to produce the Bell state

$$
\left|\Phi^{+}\right\rangle=1 / \sqrt{2}\left(|H\rangle_{a}|H\rangle_{b}+|V\rangle_{a}|V\rangle_{b}\right)
$$

for each of the pairs generated into the pairs of modes $a 1 \& b 1, a 2 \& b 2$, etc. If the back-reflection mirror is kept interferometrically stable the emission amplitudes add up coherently and the overall Hamiltonian for creat- 
ing 4 pairs via PDC can be written as

$$
\begin{gathered}
\mathcal{H} \propto\left[\sum_{m=2,4}\left(a^{\dagger} m_{H} b^{\dagger} m_{H}+a^{\dagger} m_{V} b^{\dagger} m_{V}\right)+\right. \\
\left.\sum_{n=1,3} e^{i \Delta \phi}\left(a^{\dagger} n_{H} b^{\dagger} n_{H}+a^{\dagger} n_{V} b^{\dagger} n_{V}\right)\right]^{4}+\text { h.c. }
\end{gathered}
$$

Here, $a^{\dagger} m$ and $b^{\dagger} m$ are creation operators for the forward emitted entangled photon pairs $(a 2 \& b 2$ and $a 4 \&$ $b 4)$, and $a^{\dagger} n$ and $b^{\dagger} n$ are the creation operators for the backward emitted entangled photon-pairs $(a 1 \& b 1$ and $a 3 \& b 3)$. The phase of each backward emitted photon pair is influenced by the position $x$ of the pump mirror PM with $\Delta \phi=2 \pi \frac{\Delta x}{\lambda}$ and the single-photon wavelength $\lambda$ of the downconverted photons.

To see the working mechanism of the scheme the setup can be split up in two parts (Fig. 1): on the left side the PBSs combine forward- and backward emission modes from different crystals ( $a 2 \& a 3$ and $a 4 \& a 1$ ) and on the right side forward- and backward emission modes from the same crystals are superimposed $(b 1 \& b 2$ and $b 3 \&$ b4). Assuming that the probability for the higher order emission of 5 and more photon pairs is negligible (this is typically the case in the weak interaction regime of PDC; see below), only three different emission processes can lead to the propagation of 8 photons in the 8 output modes behind the beamsplitters: either one entangled pair is emitted into each of the modes $a 1 \& b 1$, $a 2 \& b 2, a 3 \& b 3, a 4 \& b 4$ or a double-pair emission occurs into the backward modes both $a 1 \& b 1$ and $a 3$ $\& b 3$ or a double-pair emission occurs into the forward modes both $a 2 \& b 2$ and $a 4 \& b 4$. Because of the specific choice of modes to overlap at the PBSs all other possible emissions fail to provide sufficiently many photons in all output modes (For example, a three-pair emission into modes $a 2 \& b 2$ together with a single-pair emission into modes $a 4 \& b 4$ would fail to provide a coincident detection event in the output modes $\left.a 1^{\prime} \& a 4^{\prime}\right)$. The left side acts as a parity check on the photon polarization in emission modes $a 2 \& a 3$ and $a 4$ \& $a 1$. Because of the polarization-entanglement of the initially emitted pairs the overall state is projected into

$$
\begin{gathered}
|V\rangle_{a 1^{\prime}}|H\rangle_{a 2^{\prime}}|V\rangle_{a 3^{\prime}}|H\rangle_{a 4^{\prime}}|V\rangle_{b 1^{\prime}}|H\rangle_{b 2^{\prime}}|V\rangle_{b 3^{\prime}}|H\rangle_{b 4^{\prime}}+ \\
e^{i 8 \Delta \phi}|H\rangle_{a 1^{\prime}}|V\rangle_{a 2^{\prime}}|H\rangle_{a 3^{\prime}}|V\rangle_{a 4^{\prime}}|H\rangle_{b 1^{\prime}}|V\rangle_{b 2^{\prime}}|H\rangle_{b 3^{\prime}}|V\rangle_{b 4^{\prime}}+ \\
e^{i 4 \Delta \phi}\left(|H\rangle_{a 1^{\prime}}|H\rangle_{a 2^{\prime}}|H\rangle_{a 3^{\prime}}|H\rangle_{a 4^{\prime}}|H\rangle_{b 1^{\prime}}|H\rangle_{b 2^{\prime}}|H\rangle_{b 3^{\prime}}|H\rangle_{b 4^{\prime}}+\right. \\
|V\rangle_{a 1^{\prime}}|V\rangle_{a 2^{\prime}}|V\rangle_{a 3^{\prime}}|V\rangle_{a 4^{\prime}}|V\rangle_{b 1^{\prime}}|V\rangle_{b 2^{\prime}}|V\rangle_{b 3^{\prime}}|V\rangle_{b 4^{\prime}}+ \\
|H\rangle_{a 1^{\prime}}|V\rangle_{a 2^{\prime}}|V\rangle_{a 3^{\prime}}|H\rangle_{a 4^{\prime}}|H V\rangle_{b 1^{\prime}}|0\rangle_{b 2^{\prime}}|0\rangle_{b 3^{\prime}}|H V\rangle_{b 4^{\prime}}+ \\
\left.|V\rangle_{a 1^{\prime}}|H\rangle_{a 2^{\prime}}|H\rangle_{a 3^{\prime}}|V\rangle_{a 4^{\prime}}|0\rangle_{b 1^{\prime}}|V H\rangle_{b 2^{\prime}}|V H\rangle_{b 3^{\prime}}|0\rangle_{b 4^{\prime}}\right)(3)
\end{gathered}
$$

The first term stems from the two double-pair emissions into the forward modes $a 2 \& b 2$ and $a 4 \& b 4$ and hence carries a phase of $e^{i 8 \Delta \phi}$ relative to the second term that is due to the two double-pair emissions into the backward modes $a 1 \& b 1$ and $a 3 \& b 3$. The third term, with a relative phase of $e^{i 4 \Delta \phi}$, originates from the single-pair emission into all mode-pairs. Note that it has two contributions in which no photons are emitted into modes $b 1^{\prime}$ or $b 3^{\prime}$. The parity check leaves therefore only those contributions in which a detection event in $b 1^{\prime}$ and $b 3^{\prime}$ indicates with certainty that a photon each propagates in modes $b 2^{\prime}$ and $b 4^{\prime}$. In this way, the emission of a two-photon state in modes $b 2^{\prime}$ and $b 4^{\prime}$ is heralded by joint detection events in modes $a 1^{\prime}, a 2^{\prime}, a 3^{\prime}, a 4^{\prime}, b 1^{\prime}$ and $b 3^{\prime}$. By projecting each of these 6 modes into a specific linearly polarized basis, e.g. $| \pm\rangle=\frac{1}{\sqrt{2}}(|H\rangle \pm|V\rangle)$, one obtains the maximally entangled Bell state $\left|\Phi^{+}\right\rangle=\frac{1}{\sqrt{2}}\left(|H\rangle_{b 2^{\prime}}|H\rangle_{b 4^{\prime}}+\right.$ $|V\rangle_{b 2^{\prime}}|V\rangle_{b 4^{\prime}}$ ), as can be seen by projecting the state of Eq. (3) onto $|+\rangle_{a 1^{\prime}}|+\rangle_{a 2^{\prime}}|+\rangle_{a 3^{\prime}}|+\rangle_{a 4^{\prime}}|+\rangle_{b 1^{\prime}}|+\rangle_{b 3^{\prime}}$. This entangled state, $\left|\Phi^{+}\right\rangle$, can be easily converted into any other Bell state by using local operations at one of the qubits. Recent 6 -photon experiments were already able to detect 6 -fold coincidences at a rate of 40 per minute and with 2-photon visibilities beyond 90\% [7], which corresponds respectively to the expected generation rate and fidelity of the heralded pair if the same parameters were used. It is also important to note that our scheme does not need photon-number resolution in the detection process, since the scheme inherently suppresses situations in which more than one photon is emitted into each of the 6 detection modes. Furthermore, the fidelity of the created state is independent of the detection efficiency, which is an advantage over other proposals where this is not the case [8]. In our case, the fidelity will depend on the interaction parameter. Increasing the interaction will enhance the effect of stimulated emission and lead to spurious detection events that will eventually diminish the fidelity. We discuss a workaround scheme at the end of the paper. For the present discussion we focus on the situation, in which higher-order pair emissions are essentially negligible.

This scheme can easily be generalized to the heralded generation of higher photon-number entangled states. By adding additional crystals (and hence more pairs of emission modes) and by keeping the general structure (of parity-checks on the left side and projection on the right side) the above arguments hold as well. For example, one 3) additional crystal extends the scheme to 6 pairs of emission modes. Again, the only relevant emissions are those three where (1) one pair each is emitted into the pairs of modes $a 1 \& b 1, a 2 \& b 2, a 3 \& b 3, a 4 \& b 4, a 5 \& b 5, a 6 \& b 6$, or (2) a double pair each is emitted into the forward pairs of modes $a 2 \& b 2, a 4 \& b 4, a 6 \& b 6$ or (3) a double pair each is emitted into the backward pairs of modes $a 1 \& b 1, a 3 \& b 3$, 
$a 5 \& b 5$. The left-side parity check leaves the state

$$
\begin{array}{ccc} 
& |V\rangle_{a 1^{\prime}}|H\rangle_{a 2^{\prime}}|V\rangle_{a 3^{\prime}}|H\rangle_{a 4^{\prime}}|V\rangle_{a 5^{\prime}}|H\rangle_{a 6^{\prime}} & \\
|V\rangle_{b 1^{\prime}}|H\rangle_{b 2^{\prime}}|V\rangle_{b 3^{\prime}}|H\rangle_{b 4^{\prime}}|V\rangle_{b 5^{\prime}}|H\rangle_{b 6^{\prime}} & + \\
e^{i 12 \Delta \phi} & |H\rangle_{a 1^{\prime}}|V\rangle_{a 2^{\prime}}|H\rangle_{a 3^{\prime}}|V\rangle_{a 4^{\prime}}|H\rangle_{a 5^{\prime}}|V\rangle_{a 6^{\prime}} & \\
|H\rangle_{b 1^{\prime}}|V\rangle_{b 2^{\prime}}|H\rangle_{b 3^{\prime}}|V\rangle_{b 4^{\prime}}|H\rangle_{b 5^{\prime}}|V\rangle_{b 6^{\prime}} & + \\
e^{i 6 \Delta \phi}\left(|H\rangle_{a 1^{\prime}}|H\rangle_{a 2^{\prime}}|H\rangle_{a 3^{\prime}}|H\rangle_{a 4^{\prime}}|H\rangle_{a 5^{\prime}}|H\rangle_{a 6^{\prime}}\right. & \\
|H\rangle_{b 1^{\prime}}|H\rangle_{b 2^{\prime}}|H\rangle_{b 3^{\prime}}|H\rangle_{b 4^{\prime}}|H\rangle_{b 5^{\prime}}|H\rangle_{b 6^{\prime}} & + \\
|V\rangle_{a 1^{\prime}}|V\rangle_{a 2^{\prime}}|V\rangle_{a 3^{\prime}}|V\rangle_{a 4^{\prime}}|V\rangle_{a 5^{\prime}}|V\rangle_{a 6^{\prime}} & \\
\left.|V\rangle_{b 1^{\prime}}|V\rangle_{b 2^{\prime}}|V\rangle_{b 3^{\prime}}|V\rangle_{b 4^{\prime}}|V\rangle_{b 5^{\prime}}|V\rangle_{b 6^{\prime}}\right) &
\end{array}
$$

For reasons of clarity we have omitted those terms in which no photons are emitted into the modes $b^{\prime}$. Conditioning the right hand side on a 3 -fold detection event in modes $b 1^{\prime}, b 3^{\prime}, b 5^{\prime}$ results then with certainty in a 3-photon state in the undetected emission modes $b 2^{\prime}, b 4^{\prime}, b 6^{\prime}$. A projection of each of the nine detection modes $a 1^{\prime}, a 2^{\prime}, a 3^{\prime}, a 4^{\prime}, a 5^{\prime}, a 6^{\prime}, b 1^{\prime}, b 3^{\prime}, b 5^{\prime}$ into the linear polarization basis $| \pm\rangle$ finally generates a freely propagating 3-particle GHZ-state. For example, a projection onto $|+\rangle_{a 1^{\prime}}|+\rangle_{a 2^{\prime}}|+\rangle_{a 3^{\prime}}|+\rangle_{a 4^{\prime}}|+\rangle_{a 5^{\prime}}|+\rangle_{a 6^{\prime}}|+\rangle_{b 1^{\prime}}|+\rangle_{b 3^{\prime}}|+\rangle_{b 5^{\prime}}$ leads to the heralded generation of the maximally entangled state $|G H Z\rangle^{(3)}=\frac{1}{\sqrt{2}}(|H\rangle|H\rangle|H\rangle+|V\rangle|V\rangle|V\rangle)$. It is straightforward to see that this technique, applied to $2 \mathrm{n}$ emission modes (n: number of crystals), is capable of heralding n-photon GHZ-states of the type $|G H Z\rangle^{(n)}=\frac{1}{\sqrt{2}}\left(|H\rangle^{\otimes n}+|V\rangle^{\otimes n}\right)$ (Fig. 1c). The 2n-photon parity check on the left side together with the $\mathrm{n}$-fold detection in the odd modes after the PBSs on the right side conditions the emission of a n-photon state into the $\mathrm{n}$ remaining even modes on the left side. If the 3n-photon coincidence detection is performed in a specific linear polarization basis $| \pm\rangle$ the heralded state is the wanted quantum state of the GHZ-type.

We would like to note an additional feature of the presented scheme. If one decides to measure all $4 \mathrm{n}$ output ports via a $4 \mathrm{n}$-fold coincidence detection, the setup represents an interferometer with a sub shotnoise phase sensitivity. Such performance is known in the context of quantum metrology and can occur when the state propagating within the interferometer is highly non-classical. For example, path-entangled photon-number states, so-called NOON-states [18], are known to achieve Heisenberg-limited interferometric sensitivity beyond the classical shot-noise limit. In a regular Mach-Zehnder interferometer, such states are of the form $|\Psi\rangle^{(N O O N)}=1 / \sqrt{(2)}\left(|N\rangle_{A}|0\rangle_{B}+|0\rangle_{A}|N\rangle_{B}\right)$, where $A$ and $B$ are the two spatial modes of propagation within the interferometer. Here, we generate NOONstates that consist of a nonlocal $4 \mathrm{n}$-photon state [15] that is path-entangled between the $\mathrm{n}$ pairs of modes for forward emission, $a 2 \& b 2, a 4 \& b 4, \ldots$ and the $\mathrm{n}$ pairs of modes for backward emission, $a 1 \& b 1, a 3 \& b 3$, ..., i.e. $|\Psi\rangle=1 / \sqrt{(2)}\left(|4 n\rangle_{a 1 \& b 1, a 3 \& b 3, \ldots}|0\rangle_{a 2 \& b 2, a 4 \& b 4, \ldots}+\right.$ $|0\rangle_{a 1 \& b 1, a 3 \& b 3, \ldots}|4 n\rangle_{a 2 \& b 2, a 4 \& b 4, \ldots}$. Specifically, if we project all $4 \mathrm{n}$ detection modes onto linear polarizations $| \pm\rangle$ with an odd number of $|-\rangle$ projections, e.g. $|-\rangle_{a 1^{\prime}}|+\rangle_{b 1^{\prime}}|+\rangle_{a 2^{\prime}}|+\rangle_{b 2^{\prime}} \ldots|+\rangle_{a 2 n^{\prime}}|+\rangle_{b 2 n^{\prime}}$, then the $4 \mathrm{n}$-fold coincidence detection probability varies with $P_{a 1^{\prime}, b 1^{\prime}, \ldots, a n^{\prime}, b n^{\prime}} \propto 1-\cos 4 n \Delta \phi$ and the phase-noise sensitivity $\langle\Delta \phi\rangle$ scales with $\frac{1}{4 n}$ as opposed to the expected $\frac{1}{\sqrt{4 n}}$ (shot-noise) performance of a comparable "classical" interferometer (in particular, a single-photon interferometer with $2 \mathrm{n}$ independent averaging runs or an interferometer that is driven with a coherent state with $\alpha \approx 2 n$ ). Interferometers based on NOON-states have recently been demonstrated for up to 4 photons [15, 19, 20] and up to 6 ions [21, 22, 23].

As an example, consider the 8-photon interferometer described in Figure 2a, where $n=2$. Projecting each of the eight output modes onto a linear basis, $| \pm\rangle$, specifically

$$
|-\rangle_{a 1^{\prime}}|+\rangle_{a 2^{\prime}}|+\rangle_{a 3^{\prime}}|+\rangle_{a 4^{\prime}}|+\rangle_{b 1^{\prime}}|+\rangle_{b 2^{\prime}}|+\rangle_{b 3^{\prime}}|+\rangle_{b 4^{\prime}},
$$

effectively eliminates the contributions of the emission process into each pairs of modes (3rd term in Eq. 3) and results in the maximally path entangled state of the form

$$
\begin{array}{r}
|\Psi\rangle=1 / \sqrt{2}\left(|8\rangle_{a 2^{\prime} b 2^{\prime} a 4^{\prime} b 4^{\prime}}|0\rangle_{a 1^{\prime} b 1^{\prime} a 3^{\prime} b 3^{\prime}}+\right. \\
\left.e^{i 8 \Delta \phi}|0\rangle_{a 2^{\prime} b 2^{\prime} a 4^{\prime} b 4^{\prime}}|8\rangle_{a 1^{\prime} b 1^{\prime} a 3^{\prime} b 3^{\prime}}\right) .
\end{array}
$$

These remaining contributions correspond to a $N O O N$-state, where either 8 photons are emitted via four-photon emission from each crystal into the pair of modes $a 2 \& b 2$ and $a 4 \& b 4$ or by four-photon emissions into the backward pair of modes $a 1 \& b 1$ and $a 3 \& b 3$. Thus the eight-photon detection probability in the spatially separated output modes oscillates like $P_{a 1^{\prime}, a 2^{\prime}, a 3^{\prime}, a 4^{\prime}, b 1^{\prime}, b 2^{\prime}, b 3^{\prime}, b 4^{\prime}} \propto 1-\cos (8 \Delta \phi)$ when moving the delay mirror for the UV pump beam. Finally, the generalization to an arbitrary number of $4 \mathrm{n}$ photons is equivalent to the heralding scheme above and simply consists of adding crystals and keeping the overall structure of the interferometric scheme (Fig. 2b).

Let us finally comment on the potentials and limitations of the scheme. Firstly, the setup is essentially a multi-photon interferometer in which the phase needs to be controlled with high accuracy. This poses an increasing challenge with increasing number of involved photons, since the phase-sensitivity of the state contributions scale linearly with photon number (see Eq. (3) and (4)). However, locking techniques can achieve stable interferometer controls to better than $10^{-15} \mathrm{~m}$ [24], which would allow photon numbers on the order of $10^{8}$ or larger; hence phase stability does not pose a limitation. Secondly, the scheme is based on using stimulated PDC emission in the weak interaction regime where the probability to generate an additional pair is negligible. In our case, if $(2 n+1)$ pairs would be emitted instead of the wanted $2 \mathrm{n}$, spurious coincidence events might occur without generating the heralded state. The probability to create an additional pair is on the order of the interaction parameter[25], which is 
typically between 0.01 and 0.1 [26]. However, although increasing the interaction leads to larger photon-numbers it also increases the effect of stimulated emission [27, 28]. This eventually results in the more efficient production of states with higher pair numbers and hence decreases the performance of our scheme if non photon-number resolving detectors are used. The use of photon-number resolving detectors with almost perfect quantum efficiency would overcome this deficiency and the scheme would still work.

In conclusion, we have presented a scalable scheme to herald the generation of multi-photon entangled states of, in principle, arbitrary size. The setup can also be viewed (and used) as Heisenberg-limited interferometer. For PDC in the weak interaction regime standard photon detection without number resolution is sufficient to generate high-quality GHZ-type quantum states in an event-ready manner. For the strong interaction regime, which is necessary to achieve large photon numbers [28], the use of photon-number resolving detectors allows to maintain the proposed performance.

The authors thank Kevin Resch for helpful discussions. This work was supported by the Austrian Science Foundation (FWF) under project SFB15, the European Commission, contract numbers IST-2001-38864 (RAMBOQ) and IST-015848 (QAP), the DTO-funded U.S. Army Research Office Contract No. W911NF-05-0397 and by the Alexander von Humboldt-Foundation.

[1] C. H. Bennett and G. Brassard, Proceedings of IEEE International Conference on Computers, Systems and Signa Processing, Bangalore, India pp. 175-179 (1984).

[2] N. Gisin, G. Ribordy, W. Tittel, and H. Zbinden, Rev. Mod. Phys. 74, 145 (2002).

[3] E. Knill, R. Laflamme, and G. Milburn, Nature 409, 46 (2000).

[4] D. Gottesman and I. L. Chuang, Nature 402, 390 (1999).

[5] D. E. Browne and T. Rudolph, Phys. Rev. Lett. 95, 010501 (2005).

[6] D. C. Burnham and D. L. Weinberg, Phys. Rev. Lett. 25, 84 (1970).

[7] C.-Y. Lu, X.-Q. Zhou, O. Gühne, W.-B. Gao, J. Zhang, Z.-S. Yuan, A. Goebel, T. Yang, J.-W. Pan, quant$\mathrm{ph} / 0610145$ (2006).

[8] A. B. URen, C. Silberhorn, K. Banaszek, and I. A.Walmsley, Phys. Rev. Lett. 93, 093601 (2004).

[9] M. Zukowski, A. Zeilinger, M. A. Horne, and A. K. Ekert, Phys. Rev. Lett 71, 4287 (1993).
[10] P. Kok, Ph.D. thesis, University of Wales, Bangor (2000).

[11] C. Sliwa and K. Banaszek, Phys. Rev. A 67, 030101(R) (2003).

[12] T.B. Pittman et al., IEEE J. of Selected Topics in Quant. Elect. 9, 1478 (2003).

[13] A.A. Hnilo, Phys. Rev. A 71, 033820 (2005).

[14] H.S. Eisenberg, J.F. Hodelin, G. Khoury, and D. Bouwmeester, Phys. Rev. Lett. 94, 090502(2005).

[15] P. Walther, J.-W. Pan, M. Aspelmeyer, R. Ursin, S. Gasparon, and A. Zeilinger, Nature 429, 158 (2004).

[16] J.-W. Pan, M. Daniell, S. Gasparoni, G. Weihs, and A. Zeilinger, Phys. Rev. Lett. 86, 4435 (2001).

[17] T. B. Pittman, B. C. Jacobs, and J. D. Franson, Phys. Rev. A 64, 062311 (2001).

[18] P. Kok, Phys. Rev. A 63, 063407 (2001).

[19] J. G. Rarity, P. R. Tapster, E. Jakeman, T. Larchuk, R. A. Campos, M. C. Teich, and B. E. A. Saleh, Phys. Rev. Lett. 65, 1348 (1990).

[20] M. W. Mitchell, J. S. Lundeen, and A. M. Steinberg, Nature 429, 161 (2004).

[21] C. A. Sackett, D. Kielpinski, B. E. King, C. Langer, V. Meye, C. J. Myatt, M. Rowe, Q. A. Turchette, W. M. Itano, D. J. Wineland, et al., Nature 404, 256 (2000).

[22] D. Leibfried, M. D. Barrett, T. Schaetz, J. Britton, J. Chiaverin, W. M. Itano, J. D. Jost, C. Langer, and D. J. Wineland, Science 304, 1476 (2004).

[23] D. Leibfried, E. Knill, S. Seidelin, J. Britton, R. B. Blakestad, J. Chiaverini, D. B. Hume, W. M. Itano, J. D. Jost, C. Langer, et al., Nature 438, 639 (2005).

[24] T. Briant, P.-F. Cohadon, A. Heidmann, M. Pinard, Proc. EQEC 03, 327 (2003).

[25] P. Kok and S. L. Braunstein, Phys. Rev. A 61, 042304 (2000).

[26] R. Kaltenbaek, B. Blauensteiner, M. Zukowski, M. Aspelmeyer, and A. Zeilinger, to be published (2005).

[27] A. Lamas-Linares, J. C. Howell, and D. Bouwmeester, Nature 412, 887 (2001).

[28] H. S. Eisenberg, G. Khoury, G. Durkin, C. Simon, and D. Bouwmeester, Phys. Rev. Lett. 93, 193901 (2004).

Fig. 1. Schematic setup for the heralded preparation of multi-photon maximally entangled states. a Conditional generation of two-photon Bell-states. b Conditional generation of three-photon GHZ-states. c Conditional generation of multi-photon states of the GHZ-type.

Fig. 2. Multi-photon interferometer for quantum metrology. a 8-photon path-entangled NOON-states can be generated by performing a polarization measurement in all of the output modes in the linear polarization basis $| \pm\rangle$ where the number of $|-\rangle$ projections is odd. This results in pure 8-photon interference due to projection onto the (non-local) path-entangled 8-photon state $|\Psi\rangle=1 / \sqrt{2}\left(|8\rangle_{a 2 b 2 a 4 b 4}|0\rangle_{a 1 b 1 a 3 b 3}+|0\rangle_{a 2 b 2 a 4 b 4}|8\rangle_{a 1 b 1 a 3 b 3}\right)$ . b Generalized scheme for 4 n-photon NOON states. 